\title{
Maximum-entropy meshfree method for incompressible media problems
}

\author{
A. Ortiz ${ }^{*, a}$, M. A. Puso ${ }^{b}$, N. Sukumar ${ }^{\mathrm{a}}$ \\ ${ }^{a}$ Department of Civil and Environmental Engineering, University of California, One \\ Shields Avenue, Davis, CA 95616, U.S.A. \\ ${ }^{b}$ Lawrence Livermore National Laboratory, P.O. Box 808, Livermore, CA 94551, U.S.A.
}

\section{Abstract}

A novel maximum-entropy meshfree method that we recently introduced in Ref. [1] is extended to Stokes flow in two dimensions and to three-dimensional incompressible linear elasticity. The numerical procedure is aimed to remedy two outstanding issues in meshfree methods: the development of an optimal and stable formulation for incompressible media, and an accurate cell-based numerical integration scheme to compute the weak form integrals. On using the incompressibility constraint of the standard $u-p$ formulation, a $u$-based formulation is devised by nodally averaging the hydrostatic pressure around the nodes. A modified Gauss quadrature scheme is employed, which results in a correction to the stiffness matrix that alleviates integration errors in meshfree methods, and satisfies the patch test to machine accuracy. The robustness and versatility of the maximum-entropy meshfree method is demonstrated in three-dimensional computations using tetrahedral background meshes for integration. The meshfree formulation delivers optimal

\footnotetext{
*Corresponding author

Email address: alortiz@ucdavis.edu (A. Ortiz)
} 
rates of convergence in the energy and $L^{2}$-norms. Inf-sup tests are presented to demonstrate the stability of the maximum-entropy meshfree formulation for incompressible media problems.

Key words: elasticity, Stokes problem, volumetric locking, numerical integration, maximum-entropy principle, meshfree methods

\section{Introduction}

In the analysis of time-independent incompressible media problems, the Stokes problem provides a means to study the behavior of both solids and fluids. In solid mechanics, the Stokes equations arise in the treatment of incompressible linear elastic isotropic materials, whereas in fluid mechanics the Stokes equations emerge in the analysis of slow (typically, high-viscous) timeindependent flows. The weak form for the Stokes boundary-value problem leads to a saddle-point problem, which is identified with mixed variational formulations $[2,3]$. In the saddle-point problem, mathematical relations need to be fulfilled between the approximations chosen for the displacement (or velocity) and pressure fields - not all choices for these approximation spaces lead to stability. The stability of such mixed formulations is characterized by the celebrated Ladyzhenskaya-Babuška-Brezzi (LBB) inf-sup condition $[2,4,5]$. The analytical proof to establish LBB-stability is either cumbersome [6] or impossible to accomplish for distorted finite elements [7], which has led to the development of other approaches that by-pass the inf-sup condition $[8,9]$. Alternatively, a numerical test to verify the inf-sup condition has been developed [7], which can be readily performed over any finite element discretization. Finite element spaces for the displacement (or velocity) and pressure 
that pass the numerical (Chapelle-Bathe) inf-sup test are likely to satisfy its analytical counterpart [10].

The finite element literature is replete with use of mixed formulations and their stability analysis for incompressible media problems (for example, see Refs. [11, 12]). The performance and comparison among several stabilization procedures for finite elements is provided in Ref. [13]. However, less attention has been given to meshfree methods. In solid mechanics, displacement-pressure mixed formulations have been adopted for different meshfree methods in Refs. $[14,15,16,17]$ among others. In fluid mechanics, Desimone et al. [18] formulated a velocity-pressure mixed formulation using moving least-squares approximation, and a LBB-stable meshfree procedure that combines boundary integral formulation with moving leastsquares approximation was employed by Li and Zhu [19] and Li [20] in a Galerkin velocity-pressure mixed formulation. Huerta et al. [21] devised an element-free Galerkin method using approximations that asymptotically become divergence-free as the discretization is refined. Other approaches where the LBB condition does not appear as a stability criterion have also been studied with meshfree methods [22, 23, 24, 25, 26, 27]

The study of stability in meshfree mixed formulations is rendered difficult due to the rational form of meshfree basis functions and the absence of an element structure in the construction of the meshfree approximation. For instance, Dolbow and Belytschko [14] and De and Bathe [15] emphasize the difficulty in obtaining an analytical proof to the inf-sup condition and in passing the inf-sup test. A stable meshfree formulation for incompressible media based on mixed formulations is, in general, an outstanding issue in 
meshfree methods. A few instances of meshfree methods that violate the LBB condition have also been developed within the framework of stabilized methods $[28,29,30]$.

Galerkin-based meshfree methods are based on rational (non-polynomial) basis functions whose supports do not coincide with the union of background cells that are employed in the numerical integration. Due to the latter, inaccuracies in the numerical integration of weak form integrals appear. The numerical integration issue was first addressed by Dolbow and Belytschko [31]. To alleviate integration errors, they suggested the use of integration cells aligned with the support of basis functions. The aforementioned procedure is computationally expensive; instead, higher-order Gauss quadrature is usually employed. A theoretical study on the influence of numerical quadrature errors in meshfree methods was recently put forth by Babuška et al. [32]. In Ref. [32] a recipe to correct the computed stiffness matrix is proposed to eliminate the inaccuracy in the numerical integration. In Ortiz et al. [1], we introduced a modified integration scheme which reduced integration errors in general and suppressed them entirely for the constant strain patch test.

In this paper, we extend the Galerkin-based meshfree method presented in Ref. [1] to two-dimensional Stokes flow and to three-dimensional analysis of incompressible elastic solids. In a nutshell, the method consists of developing a displacement or velocity-based formulation by nodally averaging the hydrostatic pressure around the nodes in the divergence-free constraint. Additionally, numerical inf-sup tests are presented to establish the stability of the formulation, and optimal convergence in energy- and $L^{2}$-norms is established. The remainder of this paper is organized as follows. In Section 2, 
a short introduction to maximum-entropy basis functions is presented. In Section 3, the new meshfree formulation for incompressible problems is developed, and in Section 4, the inf-sup test is described for the maximumentropy meshfree method. Numerical examples are presented in Section 5 for benchmark linear problems in both solids and fluids to demonstrate the effectiveness of the proposed procedure for time-independent incompressible media problems. We also demonstrate that the meshfree formulation passes the Chapelle-Bathe inf-sup test [7] on several benchmark problems. Finally, we conclude with some final remarks in Section 6 .

\section{Maximum-entropy basis functions}

In three dimensions, the constant and linear reproducing conditions, namely, $\sum_{a=1}^{n} \phi_{a}(\mathbf{x})=1$ and $\sum_{a=1}^{n} \phi_{a}(\mathbf{x}) \mathbf{x}_{a}=\mathbf{x}$ do not prescribe unique basis functions for $n>4$ (more unknowns than linear equations). On viewing basis functions as discrete probabilities, it is suitable to apply the principle of maximum entropy [33] to find the least-biased probability distribution. The Shannon entropy is used by Sukumar [34] to construct max-ent basis functions on polygons, whereas a modified entropy functional for meshfree methods is proposed by Arroyo and Ortiz [35]. A generalization of the entropy functional of Ref. [35] is further developed in Sukumar and Wright [36] on using the notion of a 'prior' within the Shannon-Jaynes entropy functional. We adopt the latter approach in this paper. Although any meshfree basis function can be employed in our formulation, the weak Kronecker-delta property that max-ent basis functions posses on the boundary [35] makes them appealing for a meshfree method since it enables the direct imposition of 
essential boundary conditions as in finite elements ${ }^{1}$.

Consider a weight function $w_{a}(\mathbf{x})$ associated with node $a$. On using the Shannon-Jaynes entropy functional, the set of max-ent basis functions $\left\{\phi_{a}(\mathbf{x}) \geq 0\right\}_{a=1}^{n}$ is obtained via the solution of the following convex optimization problem:

$$
\max _{\phi \in \mathbb{R}_{+}^{n}}-\sum_{a=1}^{n} \phi_{a}(\mathbf{x}) \ln \left(\frac{\phi_{a}(\mathbf{x})}{w_{a}(\mathbf{x})}\right)
$$

subject to the linear reproducing conditions:

$$
\sum_{a=1}^{n} \phi_{a}(\mathbf{x})=1, \quad \sum_{a=1}^{n} \phi_{a}(\mathbf{x}) \tilde{\mathbf{x}}_{a}=\mathbf{0}
$$

where $\tilde{\mathbf{x}}_{a}=\mathbf{x}_{a}-\mathbf{x}$ are shifted nodal coordinates and $\mathbb{R}_{+}^{n}$ is the non-negative orthant. If $w_{a}(\mathbf{x})=1$ for all $a$, then the Shannon entropy functional, $-\sum_{a} \phi_{a} \ln _{a}$, is recovered. In practice, any weight function that is compactlysupported and at least $C^{0}$-continuous may be used. Typical weight functions are smooth Gaussian radial basis functions [35]

$$
w_{a}(\mathbf{x})=\exp \left(-\beta_{a}\left\|\mathbf{x}_{a}-\mathbf{x}\right\|^{2}\right)
$$

and $C^{2}$ quartic polynomials [40]

$$
w_{a}(q)=\left\{\begin{array}{lr}
1-6 q^{2}+8 q^{3}-3 q^{4} & 0 \leq q \leq 1 \\
0 & q>1
\end{array}\right.
$$

\footnotetext{
${ }^{1}$ Most available meshfree basis functions would typically not vanish on the boundary and as such special procedures are needed to enforce essential boundary conditions [37, $38,39]$.
} 
where $\beta_{a}=\gamma / h_{a}^{2} ; \gamma$ is a parameter that controls the support-width of the basis function at node $a$; and $h_{a}$ is a characteristic nodal spacing that may be distinct for each node $a$. In $n$ dimensions, we set $h_{a}$ as the distance to the $n$ nearest neighbor from node $a$. For the quartic polynomial, $q=\left\|\mathbf{x}_{a}-\mathbf{x}\right\| / \rho_{a}$ and $\rho_{a}=\gamma h_{a}$ is the radius of the basis function support at node $a$. In a recent study on max-ent meshfree methods [41], it has been shown that substantial improvements in accuracy are realized by letting the supportwidth parameters as unknowns and solving for them through the variational structure (minimizing principle) of the continuum problem.

On using the procedure of Lagrange multipliers, the solution of the variational statement $(1)$ is [36]:

$$
\phi_{a}(\mathbf{x})=\frac{Z_{a}\left(\mathbf{x} ; \boldsymbol{\lambda}^{*}\right)}{Z\left(\mathbf{x} ; \boldsymbol{\lambda}^{*}\right)}, \quad Z_{a}\left(\mathbf{x} ; \boldsymbol{\lambda}^{*}\right)=w_{a}(\mathbf{x}) \exp \left(-\boldsymbol{\lambda}^{*} \cdot \tilde{\mathbf{x}}_{a}\right)
$$

where the partition function $Z\left(\mathbf{x} ; \boldsymbol{\lambda}^{*}\right)=\sum_{b} Z_{b}\left(\mathbf{x} ; \boldsymbol{\lambda}^{*}\right)$, and in three dimensions $\tilde{\mathbf{x}}_{a}=\left[\begin{array}{lll}\tilde{x}_{a} & \tilde{y}_{a} & \tilde{z}_{a}\end{array}\right]^{\mathrm{T}}$ and $\boldsymbol{\lambda}^{*}=\left[\begin{array}{lll}\lambda_{1}^{*} & \lambda_{2}^{*} & \lambda_{3}^{*}\end{array}\right]^{\mathrm{T}}$. In (3), the Lagrange multiplier vector $\boldsymbol{\lambda}^{*}$ is the minimizer of the dual of the optimization problem posed in $(1)$

$$
\boldsymbol{\lambda}^{*}=\arg \min _{\boldsymbol{\lambda} \in \mathbb{R}^{3}} \ln Z(\mathbf{x} ; \boldsymbol{\lambda})
$$

which gives rise to the following system of nonlinear equations:

$$
\mathbf{f}(\boldsymbol{\lambda})=\nabla_{\boldsymbol{\lambda}} \ln Z(\boldsymbol{\lambda})=-\sum_{a}^{n} \phi_{a}(\mathbf{x}) \tilde{\mathbf{x}}_{a}=\mathbf{0}
$$

where $\nabla_{\boldsymbol{\lambda}}$ stands for the gradient with respect to $\boldsymbol{\lambda}$. Once the converged $\boldsymbol{\lambda}^{*}$ is found, the basis functions are computed from (3) and the gradient of the basis functions is [40]

$$
\nabla \phi_{a}=\phi_{a}\left\{\tilde{\mathbf{x}}_{a} \cdot\left[(\mathbf{H})^{-1}-(\mathbf{H})^{-1} \cdot \mathbf{A}\right]+\frac{\nabla w_{a}}{w_{a}}-\sum_{b=1}^{n} \phi_{b} \frac{\nabla w_{b}}{w_{b}}\right\}
$$


where

$$
\mathbf{A}=\sum_{b=1}^{n} \phi_{b} \tilde{\mathbf{x}}_{b} \otimes \frac{\nabla w_{b}}{w_{b}}
$$

and $\mathbf{H}$ is the Hessian matrix defined by

$$
\mathbf{H}=\nabla_{\boldsymbol{\lambda}} \mathbf{f}=\nabla_{\boldsymbol{\lambda}} \nabla_{\boldsymbol{\lambda}} \ln Z=\sum_{b=1}^{n} \phi_{b} \tilde{\mathbf{x}}_{b} \otimes \tilde{\mathbf{x}}_{b}
$$

In Fig. 1, plots of a max-ent basis function computed with a Gaussian prior for various support-width parameter $\gamma$ are illustrated for two dimensions. For $\gamma=2$, plots of the Gaussian weight function and the corresponding max-ent basis function along with their derivatives are shown in Fig. 2. For the Gaussian prior, (6a) reduces to [35]

$$
\nabla \phi_{a}=\phi_{a} \mathbf{H}^{-1} \cdot \tilde{\mathbf{x}}_{a}
$$

\section{Governing equations and variational formulation}

\subsection{Strong form}

Consider a body defined by an open bounded domain $\Omega \subset \mathbb{R}^{n}(n=$ $\{2,3\})$ with boundary $\Gamma$ such that $\Gamma=\Gamma_{u} \cup \Gamma_{t}$ and $\Gamma_{u} \cap \Gamma_{t}=\emptyset$. A nearlyincompressible linear isotropic elastic solid or fluid under static loads and no body force is governed by the following equations [42]:

$$
\begin{aligned}
\boldsymbol{\nabla} \cdot \boldsymbol{\sigma}=0 & \text { in } \Omega \\
\boldsymbol{\nabla} \cdot \mathbf{u}+\frac{p}{\eta}=0 & \text { in } \Omega
\end{aligned}
$$




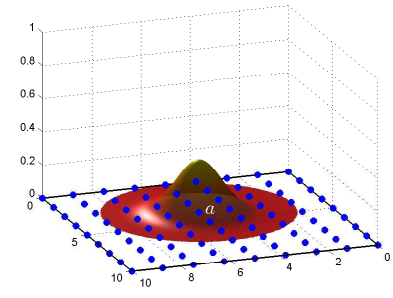

(a)

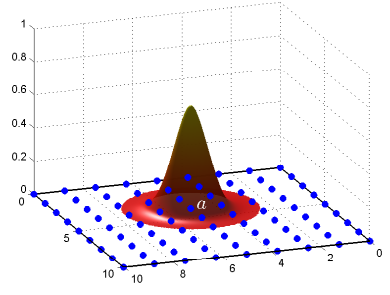

(b)

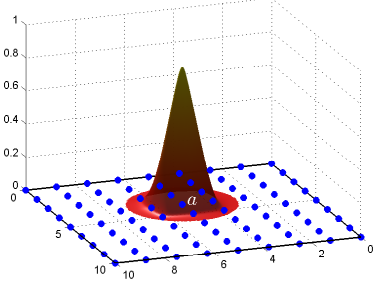

(c)

Fig. 1: Plots of a maximum-entropy basis function computed with a Gaussian prior for three values of $\gamma$. Note that the locality of the basis function is affected but it always vanishes on the boundary. (a) $\gamma=1$; (b) $\gamma=2$; and (c) $\gamma=3$.

and the following essential (displacement or velocity) and natural (traction) boundary conditions imposed on $\Gamma_{u}$ and $\Gamma_{t}$, respectively:

$$
\begin{aligned}
& \mathbf{u}=\overline{\mathbf{u}} \quad \text { on } \Gamma_{u} \\
& \boldsymbol{\sigma} \cdot \mathbf{n}=\overline{\mathbf{t}} \quad \text { on } \Gamma_{t}
\end{aligned}
$$

where the Cauchy stress tensor $\boldsymbol{\sigma}$ is related to the strain tensor $\boldsymbol{\varepsilon}$ and the pressure parameter $p$ by the following isotropic linear elastic constitutive relation:

$$
\boldsymbol{\sigma}(\mathbf{u}, p)=-p \mathbf{I}+2 \mu \boldsymbol{\varepsilon}(\mathbf{u})
$$

In (8) $\eta$ and $\mu$ are identified with the first and second Lamé parameters of the solid, whereas a penalty parameter and the dynamic viscosity of the fluid, respectively. The penalty parameter is usually taken as $\eta \sim 10^{7} \mu$ [42]. The kinematic relation between the strain tensor $\varepsilon$ and the displacement or 




(a)

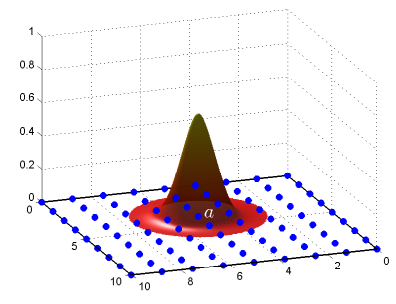

(d)

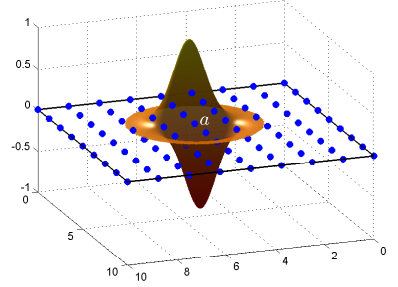

(b)

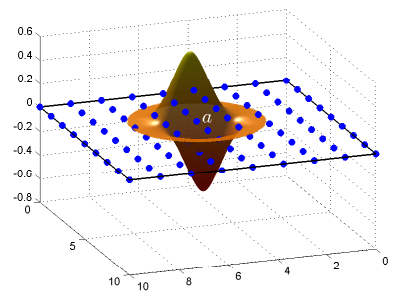

(e)

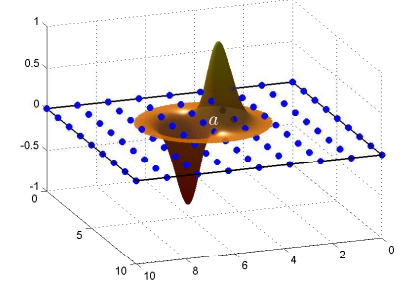

(c)

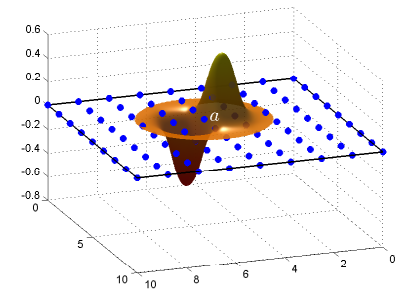

(f)

Fig. 2: Plots of Gaussian prior $(\gamma=2)$ and maximum-entropy basis function and their derivatives for node $a$. Note that $w_{a}\left(\mathbf{x}_{a}\right)=1$, but $\phi_{a}\left(\mathbf{x}_{a}\right) \neq 1$, and hence the interior basis function $\phi_{a}$ does not satisfy the Kronecker-delta property. The smoothness of the basis function and its derivatives are inherited from the Gaussian prior. (a) Gaussian prior, $w_{a}$; (b) $\partial w_{a} / \partial x$; (c) $\partial w_{a} / \partial y ;(\mathrm{d}) \phi_{a} ;$ (e) $\partial \phi_{a} / \partial x$; and (f) $\partial \phi_{a} / \partial y$. 
velocity vector $\mathbf{u}$ is:

$$
\varepsilon=\frac{1}{2}[\mathbf{u} \otimes \nabla+\nabla \otimes \mathbf{u}]
$$

\subsection{Weak form}

For Galerkin-based mixed formulations with $\mathbf{u}, \mathbf{v}$ as the trial and test displacement (or velocity) functions, and $p, q$ as the trial and test hydrostatic pressure functions, the weak form of (8) reads [42]:

$$
\begin{aligned}
& \text { Find } \mathbf{u} \in \mathscr{U} \text { and } p \in \mathscr{P} \text { such that } \\
& a(\mathbf{u}, \mathbf{v})-b(p, \mathbf{v})=(\mathbf{t}, \mathbf{v}) \quad \forall \mathbf{v} \in \mathscr{V} \\
& b(q, \mathbf{u})+\frac{1}{\eta}(p, q)=0 \quad \forall q \in \mathscr{P}
\end{aligned}
$$

where $\mathscr{U} \subset \mathbf{H}^{1}(\Omega), \mathscr{V} \equiv \mathbf{H}_{0}^{1}(\Omega)$ and $\mathscr{P} \equiv L_{0}^{2}(\Omega)$ are the usual Sobolev spaces. The bilinear forms $a(\cdot, \cdot)$ and $b(\cdot, \cdot)$ are given by

$$
\begin{gathered}
a(\mathbf{u}, \mathbf{v})=2 \mu \int_{\Omega} \varepsilon(\mathbf{u}): \varepsilon(\mathbf{v}) d \Omega \\
b(q, \mathbf{v})=\int_{\Omega} q \boldsymbol{\nabla} \cdot \mathbf{v} d \Omega
\end{gathered}
$$

whereas the linear form $(\mathbf{t}, \cdot)$ is

$$
(\mathbf{t}, \mathbf{v})=\int_{\Gamma_{t}} \mathbf{t} \cdot \mathbf{v} d \Gamma
$$

\subsection{Discrete weak form}

Our objective is to obtain a formulation solely in terms of the primary variable u. To this end, we write the pressure field in terms of nodal pressure values that are obtained by volume-averaging of the divergence-free constraint in a neighborhood of a given node. This procedure has been previously 
adopted in finite element and meshfree studies [43, 44, 45, 46]. Consider the following discretizations for the displacement (or velocity) and pressure (trial and test functions) over a background mesh of triangles or tetrahedra:

$$
\begin{aligned}
& \mathbf{u}_{h}(\mathbf{x})=\sum_{a=1}^{N} \phi_{a}(\mathbf{x}) \mathbf{u}_{a}, \quad \mathbf{v}_{h}(\mathbf{x})=\sum_{a=1}^{N} \phi_{a}(\mathbf{x}) \mathbf{v}_{a} \\
& p_{h}(\mathbf{x})=\sum_{a=1}^{N} N_{a}(\mathbf{x}) p_{a}, \quad q_{h}(\mathbf{x})=\sum_{a=1}^{N} N_{a}(\mathbf{x}) q_{a}
\end{aligned}
$$

where $N=\{3,4\}, \mathbf{u}_{h} \in \mathscr{U}_{h} \in \mathscr{U}$ and $p_{h} \in \mathscr{P}_{h} \in \mathscr{P} ; \phi_{a}$ are max-ent basis functions and $N_{a}$ are standard finite element shape functions. ${ }^{2}$ We point out that in order to ensure stability of the solution [42], the displacement (or velocity) approximation is enhanced with an extra node in the interior of each triangle or tetrahedron (see Fig. 3), which bears resemblance to the so-called MINI element [47]. However, in the meshfree case the basis function related to the interior node does not necessarily vanish on the boundary of the element. On substituting (11) into the weak form (10b) and relying on the arbitrariness of nodal pressure test functions yields

$$
\sum_{b=1}^{N} \int_{\Omega} N_{a} \mathbf{m}^{\mathrm{T}} \hat{\mathbf{B}}_{b} \mathbf{u}_{b} d \Omega+\frac{1}{\eta} \sum_{b=1}^{N} \int_{\Omega} N_{a} N_{b} p_{b} d \Omega=0
$$

and performing row-sum in the pressure term leads to

$$
\sum_{b=1}^{N}\left\{\int_{\Omega} N_{a} \mathbf{m}^{\mathrm{T}} \hat{\mathbf{B}}_{b} d \Omega\right\} \mathbf{u}_{b}+\left\{\frac{1}{\eta} \int_{\Omega} N_{a} d \Omega\right\} p_{a}=0
$$

\footnotetext{
${ }^{2}$ Note that since the derivative of the pressure does not appear in the weak form, there is no need to use meshfree basis functions and hence we adopt finite element shape functions in the discretization of the pressure.
} 


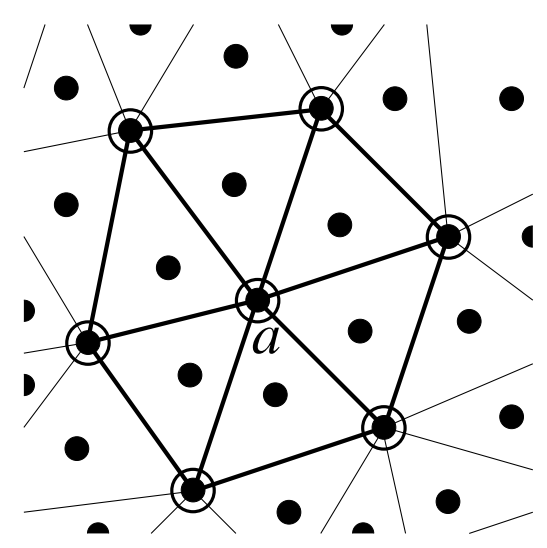

Fig. 3: Mesh to compute volume-averaged nodal pressure around a representative node $a$. Filled black circles represent displacement or velocity nodes and open circles are for pressure nodes.

Now, solving for $p_{a}$ in (13), the following volume-averaged nodal pressure is obtained [1]:

$$
p_{a}=-\eta \sum_{b=1}^{N}\left\{\frac{\int_{\bar{\Omega}} N_{a} \mathbf{m}^{\mathrm{T}} \hat{\mathbf{B}}_{b} d \Omega}{\int_{\bar{\Omega}} N_{a} d \Omega}\right\} \mathbf{u}_{b}
$$

where $\mathbf{m}^{\mathrm{T}}=\left[\begin{array}{lll}1 & 1 & 0\end{array}\right]$ in two dimensions or $\mathbf{m}^{\mathrm{T}}=\left[\begin{array}{lllll}1 & 1 & 1 & 0 & 0\end{array}\right]$ in three dimensions.

For the purpose of computation of integrals in (14), $\bar{\Omega}$ is the union of all the elements attached to node $a$, i.e., $\bar{\Omega}=\cup \Omega_{a}^{e}$. A reference mesh for our proposed method in two dimensions is illustrated in Fig. 3. In three dimensions the 3-node triangle is replaced by a 4-node tetrahedron. A similar approach but with different roots has been recently proposed by Krysl and Zhu [46] for finite elements within a nodal integration framework. In (14), $\hat{\mathbf{B}}_{a}(\mathbf{x})$ is a special form of the standard strain matrix $\mathbf{B}_{a}(\mathbf{x})$ associated with node $a$, i.e., the matrix that results from the discretization of (9) 
using $\mathbf{u}_{h}(\mathbf{x})=\sum_{a=1}^{N} \phi_{a}(\mathbf{x}) \mathbf{u}_{a}$

$$
\mathbf{B}_{a}(\mathbf{x})=\left[\begin{array}{cc}
\phi_{a, x} & 0 \\
0 & \phi_{a, y} \\
\phi_{a, y} & \phi_{a, x}
\end{array}\right]
$$

in two dimensions or

$$
\mathbf{B}_{a}(\mathbf{x})=\left[\begin{array}{ccc}
\phi_{a, x} & 0 & 0 \\
0 & \phi_{a, y} & 0 \\
0 & 0 & \phi_{a, z} \\
\phi_{a, y} & \phi_{a, x} & 0 \\
\phi_{a, z} & 0 & \phi_{a, x} \\
0 & \phi_{a, z} & \phi_{a, y}
\end{array}\right]
$$

in three dimensions, and which defines $\varepsilon_{h}=\sum_{a=1}^{N} \mathbf{B}_{a}(\mathbf{x}) \mathbf{u}_{a}$.

In Ref. [1], we have shown that the use of (15) in the numerical integration of the weak form (10) using standard (STD) Gauss quadrature may lead to inaccurate results. Similarly, the same applies for (16). The reason for such inaccuracies is well-documented in Ref. [31] and can be understood due to the following characteristic of meshfree basis functions. Meshfree basis functions are rational (non-polynomial) functions and their support do not coincide with the union of background cells that are employed in the numerical integration. When performing numerical integration of the weak form, multiplication between $\mathbf{B}_{a}$ 's for two distinct nodes arises. This in turn leads to multiplication between basis function derivatives (for instance, $\phi_{a, x} \phi_{b, y}$ ) whose support is the intersection of the support of $\phi_{a}$ and $\phi_{b}$ and as such can differ appreciably from the union of the cells used in the numerical integration. As a consequence, significant numerical errors can be expected 
from the numerical integration using (15) or (16). In an endeavor to alleviate integration errors in meshfree methods, we have devised a modified (MOD) numerical integration scheme in Ref. [1]. The procedure is summarized as follows. Let us consider the following modified strain (resemblance to assumed strain methods [48]) in a certain 3-node triangular or 4-node tetrahedral background (integration) cell:

$$
\hat{\varepsilon}=\varepsilon-\bar{\varepsilon}+\overline{\bar{\varepsilon}}
$$

where $\varepsilon$ is the standard small strain tensor, $\bar{\varepsilon}$ is the volume average strain tensor over the background cell, and $\overline{\bar{\varepsilon}}$ corresponds to $\bar{\varepsilon}$ written as a surface integral by means of Green's theorem. The corresponding equations are

$$
\begin{aligned}
& \boldsymbol{\varepsilon}=\frac{1}{2}[\mathbf{u} \otimes \boldsymbol{\nabla}+\boldsymbol{\nabla} \otimes \mathbf{u}] \\
& \bar{\varepsilon}=\frac{1}{V^{e}} \int_{\Omega^{e}} \boldsymbol{\varepsilon} d \Omega \\
& \overline{\bar{\varepsilon}}=\frac{1}{V^{e}} \int_{\Gamma^{e}} \frac{1}{2}[\mathbf{u} \otimes \mathbf{n}+\mathbf{n} \otimes \mathbf{u}] d \Gamma
\end{aligned}
$$

where (18c) is used in nodally-integrated finite element and meshfree methods [49]. In the numerical examples that are presented in this paper, we refer to the integral in (18b) as the volume integral and the integral in (18c) as the surface integral. When linearly complete finite elements are used, (18b) and (18c) yield the same result with $\bar{\varepsilon}=\overline{\bar{\varepsilon}}$, and the standard strain tensor is recovered. However, for meshfree basis functions $\bar{\varepsilon} \neq \overline{\bar{\varepsilon}}$, in general. The latter observation allows one to see $\bar{\varepsilon}-\overline{\bar{\varepsilon}}$ as a correction that is introduced into the stiffness matrix such that the integration error is reduced when the same Gauss quadrature rule is employed to integrate $\varepsilon$ as well as $\bar{\varepsilon}$. On the other hand, the evaluation of (18c) is carried out using Gauss 
quadrature along the boundary of the cell. The explicit form of $\hat{\mathbf{B}}_{a}(\mathbf{x})$ is obtained by discretizing (17) with $\mathbf{u}_{h}(\mathbf{x})=\sum_{a=1}^{N} \phi_{a}(\mathbf{x}) \mathbf{u}_{a}$, which yields

$$
\hat{\mathbf{B}}_{a}(\mathbf{x})=\mathbf{B}_{a}(\mathbf{x})-\overline{\mathbf{B}}_{a}+\overline{\overline{\mathbf{B}}}_{a}
$$

where in two and three dimensions

$$
\overline{\mathbf{B}}_{a}=\sum_{p=1}^{n} \mathbf{B}_{a}\left(\mathbf{x}_{p}\right) w_{p}
$$

whereas in two dimensions

$$
\begin{array}{r}
\overline{\overline{\mathbf{B}}}_{a}=\frac{1}{A^{e}} \sum_{L=1}^{3}\left\{\sum_{r=1}^{m} \overline{\overline{\mathbf{N}}}_{a}\left(\xi_{r}\right)\left|J\left(\xi_{r}\right)\right| w_{r}\right\} \\
\overline{\overline{\mathbf{N}}}_{a}\left(\xi_{r}\right)=\left[\begin{array}{cc}
\phi_{a} n_{x} & 0 \\
0 & \phi_{a} n_{y} \\
\phi_{a} n_{y} & \phi_{a} n_{x}
\end{array}\right]
\end{array}
$$

or in three dimensions

$$
\begin{gathered}
\overline{\overline{\mathbf{B}}}_{a}=\frac{1}{V^{e}} \sum_{L=1}^{4}\left\{\sum_{r=1}^{m} \overline{\overline{\mathbf{N}}}_{a}\left(\mathbf{x}_{r}\right) w_{r}\right\} A^{L} \\
\overline{\mathbf{N}}_{a}\left(\mathbf{x}_{p}\right)=\left[\begin{array}{ccc}
\phi_{a} n_{x} & 0 & 0 \\
0 & \phi_{a} n_{y} & 0 \\
0 & 0 & \phi_{a} n_{z} \\
\phi_{a} n_{y} & \phi_{a} n_{x} & 0 \\
\phi_{a} n_{z} & 0 & \phi_{a} n_{x} \\
0 & \phi_{a} n_{z} & \phi_{a} n_{y}
\end{array}\right]
\end{gathered}
$$

In two as well as three dimensions, $\overline{\overline{\mathbf{N}}}_{a}$ is evaluated along the boundary of the element. Note that when $\overline{\mathbf{B}}_{a}-\overline{\overline{\mathbf{B}}}_{a}=0$, the standard strain matrix $\mathbf{B}_{a}$ is recovered. 
Now, substituting (11) along with the nodal pressure expression (14) into the weak form (10a), and appealing to the arbitrariness of nodal test functions, the following discrete system of equations is obtained:

$$
\mathrm{Kd}=\mathrm{f}
$$

where $\mathbf{d}$ is the vector of nodal coefficients and

$$
\begin{aligned}
\mathbf{K}_{a b} & =\int_{\Omega} \hat{\mathbf{B}}_{a}^{\mathrm{T}} \overline{\mathbf{C}} \hat{\mathbf{B}}_{b} d \Omega-\int_{\Omega} \hat{\mathbf{B}}_{a}^{\mathrm{T}} \mathbf{m}\left\{\sum_{c=1}^{3} N_{c} \mathbf{Q}_{c b}\right\} d \Omega \\
\mathbf{f}_{a} & =\int_{\Gamma_{t}} \phi_{a} \overline{\mathbf{t}} d \Gamma
\end{aligned}
$$

with

$$
\overline{\mathbf{C}}=\left[\begin{array}{ccc}
2 \mu & 0 & 0 \\
0 & 2 \mu & 0 \\
0 & 0 & \mu
\end{array}\right]
$$

for plane strain or

$$
\overline{\mathbf{C}}=\left[\begin{array}{cccccc}
2 \mu & 0 & 0 & 0 & 0 & 0 \\
0 & 2 \mu & 0 & 0 & 0 & 0 \\
0 & 0 & 2 \mu & 0 & 0 & 0 \\
0 & 0 & 0 & \mu & 0 & 0 \\
0 & 0 & 0 & 0 & \mu & 0 \\
0 & 0 & 0 & 0 & 0 & \mu
\end{array}\right]
$$

in three dimensions and

$$
\mathbf{Q}_{c b}=-\eta\left\{\frac{\int_{\bar{\Omega}} N_{c} \mathbf{m}^{\mathrm{T}} \hat{\mathbf{B}}_{b} d \Omega}{\int_{\bar{\Omega}} N_{c} d \Omega}\right\}
$$


Note that only unknowns related to $\mathbf{u}$ appear in the system given in (20). The pressure field $p$ can be computed a posteriori from the $\mathbf{u}$ field through (14). The numerical evaluation of the integrals appearing in (20) is performed over the background mesh of triangles or tetrahedra using the modified numerical integration scheme that was described earlier.

\section{Inf-sup condition and numerical inf-sup test}

Consider the bilinear forms appearing in the weak form (10). The optimality and stability of the mixed formulation is guaranteed if the consistency of the approximation, the ellipticity of $a(\cdot, \cdot)$ on the null space of $b(\cdot, \cdot)$ and the LBB inf-sup condition $[2,4,5]$ on $b(\cdot, \cdot)$ are satisfied [10]. By construction, max-ent basis functions satisfy the linear consistency required by the weak form (10). On the other hand, if numerical integration is accurate enough - which is the case herein (see Section 3.3), the ellipticity condition is always met by displacement or velocity-pressure mixed formulations [50]. What remains to be established for the stability of the meshfree formulation is the satisfaction of the inf-sup condition $[2,4,5]$ :

$$
\inf _{q_{h} \in \mathbb{H}^{0}(\Omega)} \sup _{\mathbf{v}_{h} \in \mathbb{H}_{0}^{1}(\Omega)} \frac{\int_{\Omega}\left|q_{h} \boldsymbol{\nabla} \cdot \mathbf{v}_{h}\right| d \Omega}{\left\|q_{h}\right\|_{0}\left\|\mathbf{v}_{h}\right\|_{1}}=\alpha_{h} \geq \alpha>0
$$

holds with $\alpha$ a positive constant independent of $h$. Since in our formulation the pressure field is eliminated by writing it as a function of the displacement (or velocity) field, i.e., $q_{h} \equiv q_{h}\left(\mathbf{w}_{h}\right)$, the following equivalent form of the infsup condition is useful:

$$
\inf _{\mathbf{w}_{h} \in \mathbb{H}_{0}^{1}(\Omega)} \sup _{\mathbf{v}_{h} \in \mathbb{H}_{0}^{1}(\Omega)} \frac{\int_{\Omega}\left|q_{h}\left(\mathbf{w}_{h}\right) \boldsymbol{\nabla} \cdot \mathbf{v}_{h}\right| d \Omega}{\left\|q_{h}\left(\mathbf{w}_{h}\right)\right\|_{0}\left\|\mathbf{v}_{h}\right\|_{1}}=\alpha_{h} \geq \alpha>0
$$


The condition in (22) is verified through the numerical inf-sup test $[7,10]$. To this end, let us consider the matrix (numerical) form of (22), namely

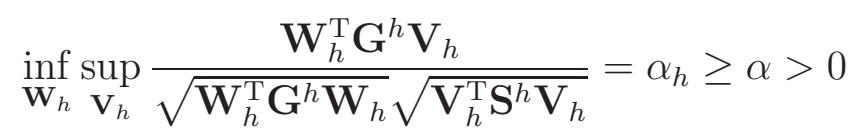

where $\mathbf{W}_{h}$ and $\mathbf{V}_{h}$ are vectors corresponding to the nodal displacement or velocity test functions $\mathbf{w}_{h}$ and $\mathbf{v}_{h}$ with

$$
\left\|q_{h}\left(\mathbf{w}_{h}\right)\right\|_{0}^{2}=\int_{\Omega}\left(q_{h}\left(\mathbf{w}_{h}\right)\right)^{2} d \Omega=\mathbf{W}_{h}^{\mathrm{T}} \mathbf{G}^{h} \mathbf{W}_{h}
$$

and

$$
\left\|\mathbf{v}_{h}\right\|_{1}^{2}=\int_{\Omega} \sum_{i, j=1}^{2}\left(\frac{\partial\left(\mathbf{v}_{h}\right)_{i}}{\partial x_{j}}\right)^{2} d \Omega=\mathbf{V}_{h}^{\mathrm{T}} \mathbf{S}^{h} \mathbf{V}_{h}
$$

In our meshfree formulation, matrices $\mathbf{G}^{h}$ and $\mathbf{S}^{h}$ in two dimensions are given by

$$
\begin{aligned}
\mathbf{G}_{a b}^{h} & =\int_{\Omega} \hat{\mathbf{B}}_{a}^{\mathrm{T}} \mathbf{m} \sum_{c=1}^{3} N_{c}\left\{\frac{\int_{\bar{\Omega}} N_{c} \mathbf{m}^{\mathrm{T}} \hat{\mathbf{B}}_{b} d \Omega}{\int_{\bar{\Omega}} N_{c} d \Omega}\right\} d \Omega \\
\mathbf{S}_{a b}^{h} & =\int_{\Omega}\left[\begin{array}{cc}
\phi_{a, x} \phi_{b, x}+\phi_{a, y} \phi_{b, y} & 0 \\
0 & \phi_{a, x} \phi_{b, x}+\phi_{a, y} \phi_{b, y}
\end{array}\right] d \Omega
\end{aligned}
$$

The numerical evaluation of the inf-sup value $\alpha_{h}$ in (23) is based on the solution of the following generalized eigenvalue problem, which is computed on a sequence of refined meshes [7, 10]:

$$
\mathbf{G}^{h} \boldsymbol{\psi}=\omega \mathbf{S}^{h} \boldsymbol{\psi}
$$

If the eigenvalues are set in increasing order, then the smallest nonzero eigenvalue $\omega_{k}$ is used to compute the numerical inf-sup value $\alpha_{h}$ as $[7,10]$

$$
\alpha_{h}=\sqrt{\omega_{k}}
$$


provided that there are no spurious pressure modes. The number of pressure modes can be anticipated from [10]

$$
k_{p m}=k-\left(n_{u}-n_{p}+1\right)
$$

where $n_{u}$ is the number of displacement or velocity degrees of freedom and $n_{p}$ the number of pressure degrees of freedom. A formulation that passes the inf-sup test must do so with $k_{p m}=0$ (no pressure modes) or if $k_{p m}>0$ (constant or spurious pressure modes), the pressure modes must be constant pressure modes as these can be removed by appropriate modification of the essential boundary conditions $[10,6]$. Hence, a formulation that is free of spurious pressure modes and does not show a decrease towards a vanishing $\alpha_{h}$ with mesh refinement is said to pass the inf-sup test. In Section 5.5, we show that the inf-sup test is passed by the maximum-entropy meshfree method for several benchmark problems.

\section{Numerical examples}

We now examine the performance of the maximum-entropy meshfree method (MEM) for three-dimensional analysis of incompressible linear elastic solids and for Stokes flow in two dimensions. In the numerical experiments, STD stands for standard Gauss quadrature (i.e., $\overline{\mathbf{B}}_{a}-\overline{\overline{\mathbf{B}}}_{a}=0$ ) and MOD for the modified integration scheme described in Section 3.3 (i.e., $\overline{\mathbf{B}}_{a}-\overline{\overline{\mathbf{B}}}_{a} \neq 0$ ). Unless stated otherwise, we use MOD with a second-order accurate scheme for both the volume and surface integrals. Low-order (4-node) tetrahedral meshes are well-known to behave poorly for the analysis of incompressible solids, which has spurred research on special finite element formulations to 
overcome this deficiency $[43,44,51,52,53,45,54,46]$. In three dimensions it is possible to obtain poor tessellations (i.e, near slivers), which can lead to inaccurate results using finite elements. In this instance, the use of meshfree basis functions appears as a more robust alternative since these basis functions do not depend on the element topology. We consider two three-dimensional benchmark problems in incompressible linear elasticity to illustrate the capability of our proposed meshfree method on unstructured tetrahedral meshes.

\subsection{Three-dimensional cantilever beam}

In this example, we study a three-dimensional cantilever beam subjected to an end load to establish the robustness of the maximum-entropy meshfree method in bending problems. The geometry, boundary and loading conditions are depicted in Fig. 4(a). The tetrahedral background mesh used in this example is illustrated in Fig. 4(b). The geometry and loading parameters are set as follows: $L=21, H=4, W=6$ and $P=50000$. The following material parameters are considered: $E=200000$ and $\nu=0.4999$. We focus on the tip deflection at point $A$ of the beam whose exact solution is -21.11 , as well as on the smoothness of the pressure field. The analysis is conducted for the MINI element and the MEM method. In the latter case we use the MOD integration technique. The corresponding numerical solutions are shown in Figs. 5(a) and 5(b) for the MINI element, whereas Figs. 5(c) and 5(d) depict the solutions for the MEM method. We note that the maximum-entropy solution is proximal to the exact one, whereas the MINI element solution behaves somewhat 'stiff' on the same mesh. Additionally, we observe that the pressure field is smoother in the maximum-entropy meshfree method than in 


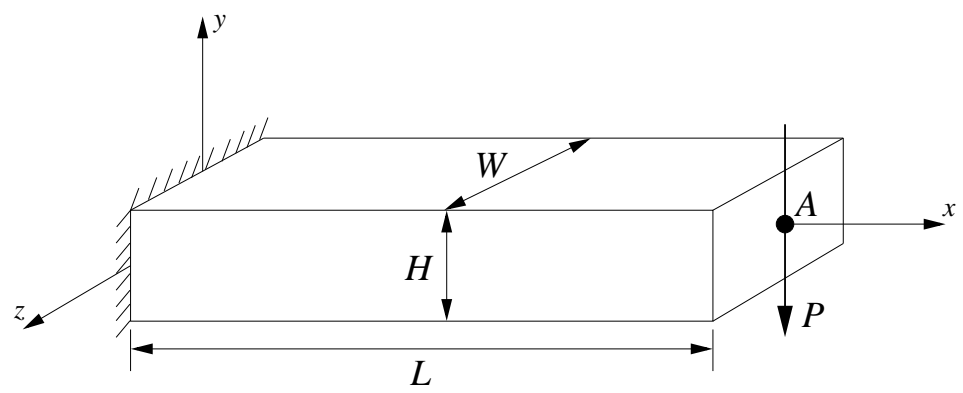

(a)



(b)

Fig. 4: Three-dimensional cantilever beam. (a) Geometry, boundary and loading conditions; and (b) background mesh for integration.

the MINI element method.

In order to demonstrate the need for the MOD integration scheme in three dimensions, we conduct the same analysis using an eight-order Gauss quadrature rule (STD integration scheme) for the MEM method. The numerical and exact tip deflections at point $A$ are summarized in Table 1. We observe that the STD scheme can not deliver the correct result, and indeed higher-order 


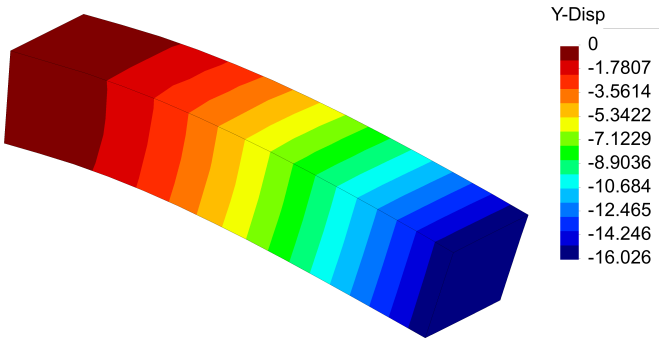

(a)



(c)

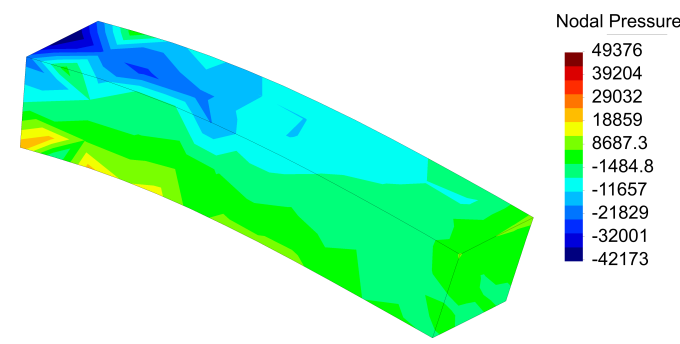

(b)

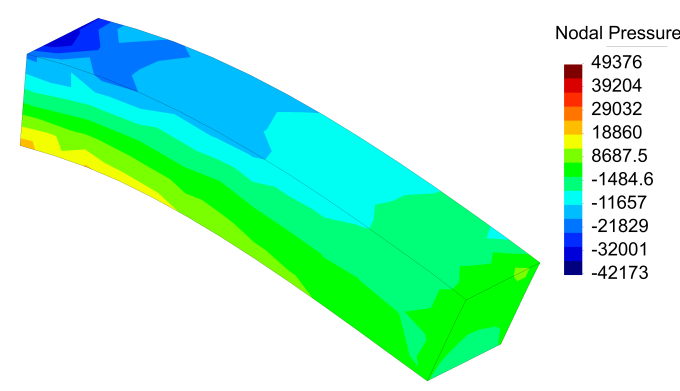

(d)

Fig. 5: Three-dimensional cantilever beam. (a) MINI element solution for vertical displacement; (b) MINI element solution for hydrostatic pressure field; (c) MEM solution for vertical displacement; and (d) MEM solution for hydrostatic pressure field.

Gauss quadrature is needed. The latter is not surprising since due to the unstructured mesh, the support of basis functions can get significantly large leading to under-integration if the accuracy of the quadrature rule employed is not sufficient. However, use of very higher-order Gauss quadrature is unappealing in a meshfree method since it imposes a computational burden on the simulations. 
Table 1: Tip deflection for the cantilever beam at point $A$.

\begin{tabular}{|c|c|c|}
\hline Method & Numerical & Exact \\
\hline MINI & -16.02 & -24.11 \\
MEM (MOD) & -24.02 & -24.11 \\
MEM (STD) & -141.99 & -24.11 \\
\hline
\end{tabular}

\subsection{Three-dimensional rigid flat punch}

In this example, we consider a simple model of three-dimensional frictionless indentation to showcase the performance of the MEM method under compressive loads. Similar benchmark problems are typically studied in two dimensions $[55,56,57]$. The geometry of the problem is depicted in Fig. 6(a). A severe constraint on allowable deformation states is introduced by fully clamping the bottom surface and the four lateral surfaces. A frictionless downward displacement of 0.15 is applied on the center of the top surface within a square area of $2 / 3 \times 2 / 3$. Due to the symmetry of the problem, only a quarter of the geometry is considered. The material parameters are set to $E=3 \times 10^{7}$ and $\nu=0.4999$. The unstructured tetrahedral background mesh shown in Fig. 6(b) is used to demonstrate the ability of the MEM method. The numerical solutions for the MINI element are presented in Figs. 7(a) and 7(b), whereas the maximum-entropy solutions are depicted in Figs. 7(c) and 7(d). In light of these results, the MEM method is clearly superior in the prediction of the displacement field and in realizing a smoother pressure solution. 


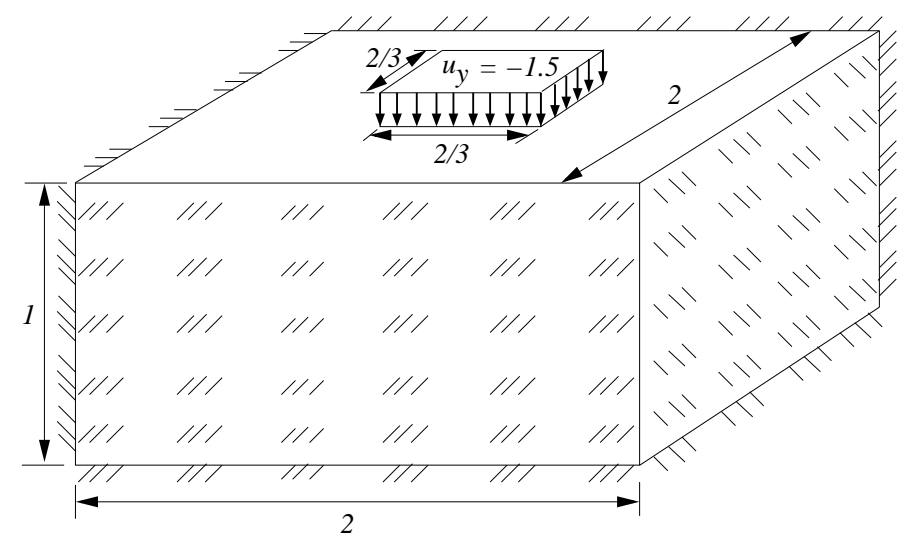

(a)

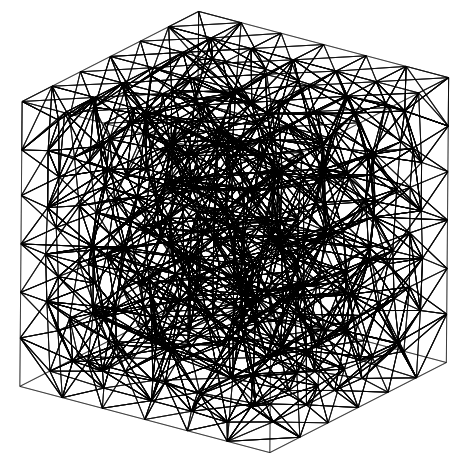

(b)

Fig. 6: Three-dimensional rigid flat punch. (a) Geometry and boundary conditions; and (b) background mesh for integration.

\subsection{Leaky-lid driven cavity flow}

The leaky-lid driven cavity flow problem is a standard benchmark to test the performance of numerical methods in incompressible flow $[42,58,59,60]$. The geometry, background mesh and prescribed velocity along the boundary of the domain are depicted in Fig. 8. Max-ent basis functions are used with a support-width parameter $\gamma=2.0$ for the Gaussian prior.

In Fig. 9, the numerical velocity and hydrostatic pressure fields for the 


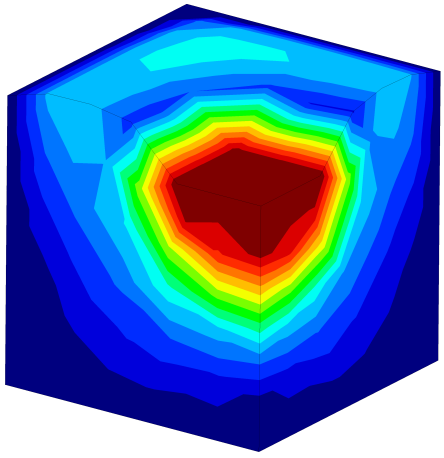

(a)

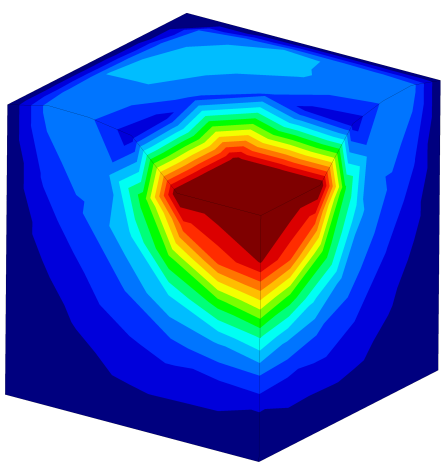

(c)


Nodal Pressure

$6.8928 \mathrm{e}+06$
$6.0212 \mathrm{e}+06$

$6.0212 \mathrm{e}+06$
$5.1495 \mathrm{e}+06$

$4.2779 \mathrm{e}+06$

$3.4062 \mathrm{e}+06$

$2.5346 \mathrm{e}+06$

$1.663 \mathrm{e}+06$

$1.9131 \mathrm{e}+05$

$-8.5197 \mathrm{e}+05$

(b)


Nodal Pressure

- $6.8928 \mathrm{e}+06$

$6.0212 \mathrm{e}+06$

$5.1495 \mathrm{e}+06$

$3.47930+06$

$3.40630+06$

$1.6630+06$

$1.663 \mathrm{e}+06$

$7.9132 e+05$
-80325

-80325
$-9.5197 e+05$

(d)

Fig. 7: Three-dimensional rigid flat punch. (a) MINI element solution for displacement field; (b) MINI element solution for hydrostatic pressure field; (c) MEM solution for displacement field; and (d) MEM solution for hydrostatic pressure field.

MINI element and MEM formulation are compared. We observe that the velocity field is quite similar for both approximations and that they are in agreement with the numerical results of Ref. [59]. We also observe in Fig. 10 a good match between the velocity of the MINI and MEM solutions. However, the MEM method better predicts the hydrostatic pressure field with a smoother solution throughout the domain. This behavior is also confirmed 




Fig. 8: Leaky-lid driven cavity flow. Geometry, mesh and boundary conditions.

by the results shown in Fig. 11, where the nodal pressure is plotted for two background meshes along line $Q-Q$. The first one, a coarser mesh of $12 \times 12$ divisions and the second one, a finer mesh of $24 \times 24$ divisions shown in Fig. 8 . On the finer mesh, the MINI element solution drifts away from the smooth MEM solution, and the former also has some oscillations. The situation is still worse for the MINI element in the coarser mesh, whereas the MEM solution on both meshes is similar and they are in agreement with the results of Ref. [59].

\subsection{Colliding flow}

We consider a simple model of colliding flow, which is a well-known and standard benchmark test problem (for example, see Refs. [61, 62]). The ge- 




(a)

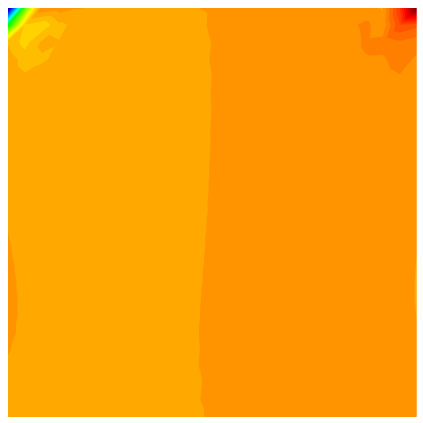

(c)
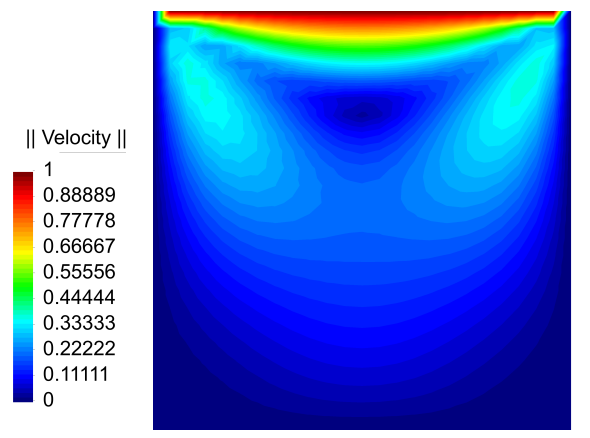

(b)
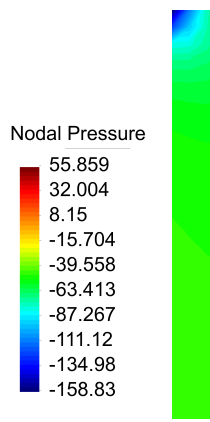

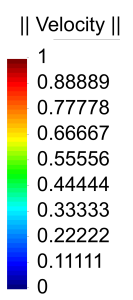

Nodal Pressure

24.184

18.542

12.901

7.2599

1.6187

$-4.0225$

-4.0225
-9.6637

$-15.305$

$-20.946$

-20.946
-26.587

(d)

Fig. 9: Leaky-lid driven cavity flow. (a) MINI element solution for velocity field, (b) MEM solution for velocity field, (c) MINI element solution for hydrostatic pressure field; and (d) MEM solution for hydrostatic pressure field.

ometry, a typical background mesh and boundary conditions are shown in Fig. 12. We study the convergence of the MEM formulation with successive nodal refinement and the influence of the numerical integration scheme employed. To this end, we use the energy norm of the error and the $L^{2}$-norm of both the velocity and pressure error, together with the following analytical 


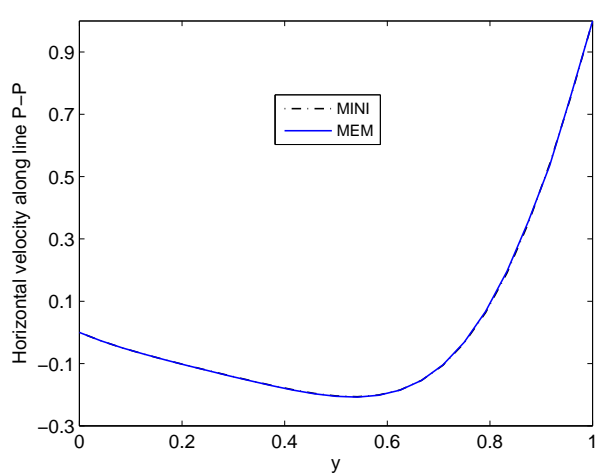

(a)



(b)

Fig. 10: Leaky-lid driven cavity flow. Nodal velocity measured along lines (a) $P-P$ and (b) $Q-Q$.



(a)

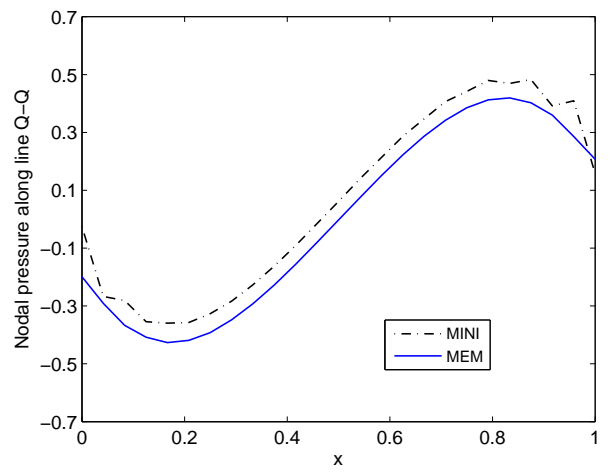

(b)

Fig. 11: Leaky-lid driven cavity flow. Nodal pressure measured along line $Q-Q$ (a) for $12 \times 12$ mesh and (b) for $24 \times 24$ mesh. 
solution [62]:

$$
\begin{aligned}
& u=20(x-1)(y-1)^{3} \\
& v=5(x-1)^{4}-5(y-1)^{4} \\
& p=60(x-1)^{2}(y-1)-20(y-1)^{3}
\end{aligned}
$$

Eqs. (29a) and (29b) are used as essential boundary conditions along the boundary of the domain. Max-ent basis functions are used with a supportwidth parameter $\gamma=1.5$ for the quartic prior. The velocity and pressure fields for the reference mesh of Fig. 12 are depicted in Fig. 13 for the MINI element and MEM solutions. Firstly, we observe that for both approximations, a refined mesh is needed to obtain an acceptable solution - the analytical solution is quartic and both approximations are linearly complete. Secondly, the MINI element and MEM solutions for the velocity field are quite similar. Thirdly, a smooth pressure field is obtained for the MEM near the corners of the domain, whereas the MINI element solution has some oscillations.

To assess the influence of numerical integration, a study of the MEM method with STD and MOD schemes is conducted (see Section 3.3). The numerical results are presented in Fig. 14. As we pointed out in Section 3.3, due to numerical integration errors in meshfree methods, low-order standard Gauss quadrature can not deliver accurate numerical solutions with optimal rates of convergence. Improved accuracy and better rate of convergence in $L^{2}$-norm are obtained with a 6-point rule, and a 12-point rule is able to deliver about the same accuracy as the modified integration scheme (Figs. 14(a) and 14(b)). It is noteworthy to point out that for the energy norm curves shown in Fig. 14(c), the STD scheme with a 12-point rule results 


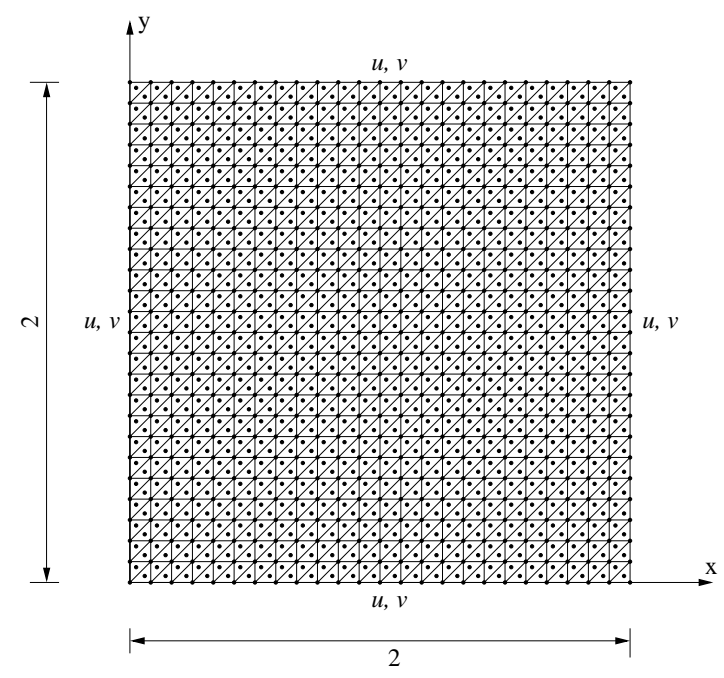

Fig. 12: Colliding flow. Geometry, mesh and boundary conditions.

in a sudden change in the slope of the curve with mesh refinement (quadrature error is not sufficiently smaller than the approximation error), whereas the MOD scheme consistently yields the optimal rate of convergence.

Finally, the accuracy and rate of convergence of the MINI element and the maximum-entropy meshfree method are compared in Fig. 15. We observe that the MINI element and MEM solutions have the optimal rate of convergence in the energy and $L^{2}$-norms, but that the MEM formulation can deliver more accurate results.

\subsection{Inf-sup tests}

The numerical inf-sup test described in Section 4 has been assessed for meshfree methods $[14,15]$. Here, we apply the inf-sup test on three problems: leaky-lid driven cavity flow, Poiseuille flow, and a square domain (the same used for the cavity flow) with zero-velocity imposed along the boundary. When a vanishing velocity is imposed along the boundary of the domain, a 


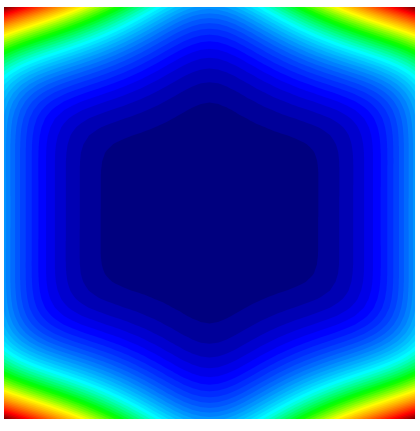

(a)

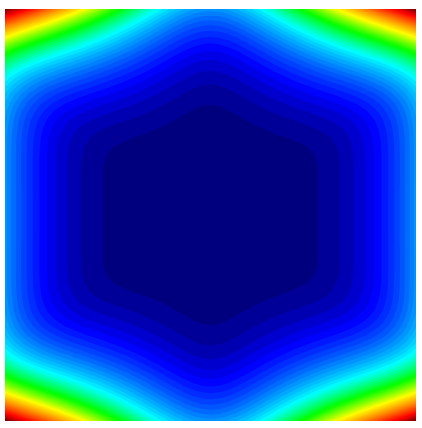

(c)

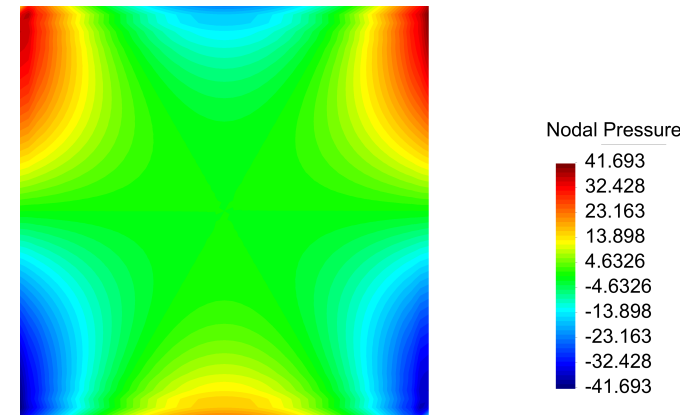

(b)

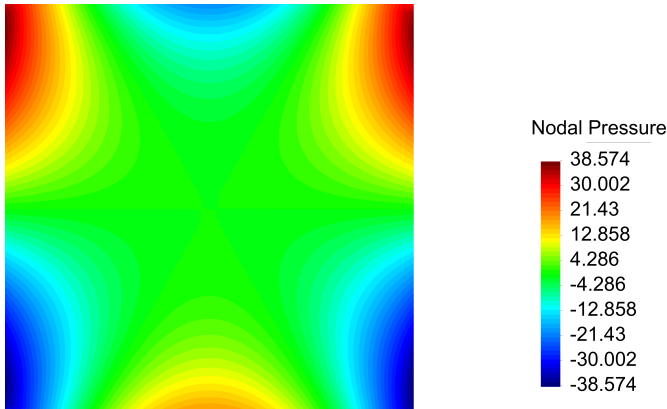

$(\mathrm{d})$

Fig. 13: Colliding flow. (a) MINI element solution for velocity field, (b) MINI element solution for hydrostatic pressure field, (c) MEM solution for velocity field; and (d) MEM solution for hydrostatic pressure field.

zero pressure field must be obtained everywhere. Otherwise, the formulation would suffer from spurious pressure modes [6]. In order to compute the numerical inf-sup value, four nodal discretizations are considered in each problem. The background meshes are shown in Fig. 16. Numerical inf-sup values are presented in Table 2. From Table 2, we observe that for all the tests the numerical inf-sup values converge to a value that is bounded away from zero with successive mesh refinements. Since the whole boundary has been imposed with essential boundary conditions for the leaky-lid driven 


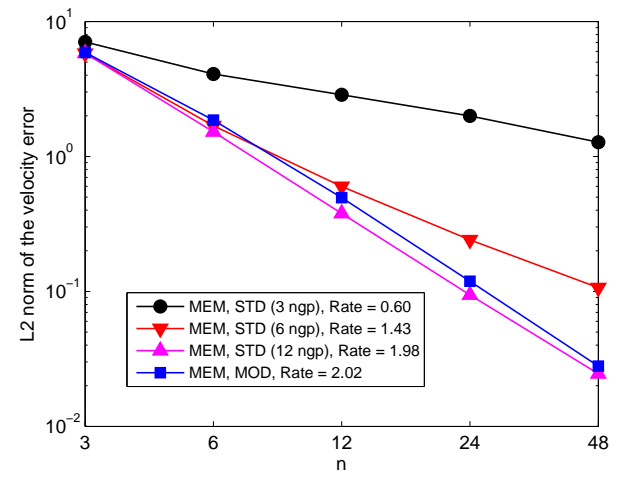

(a)

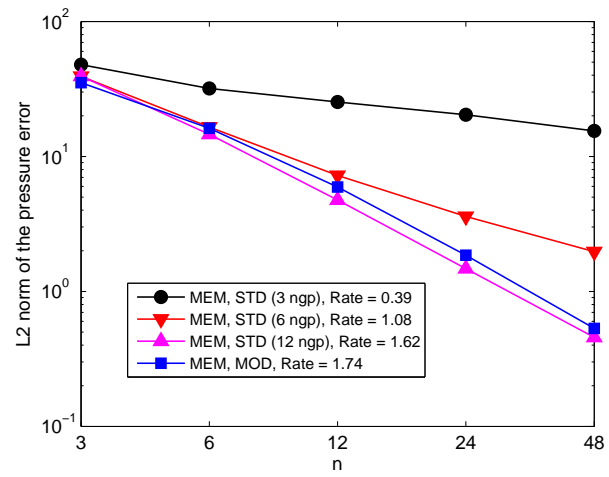

(b)

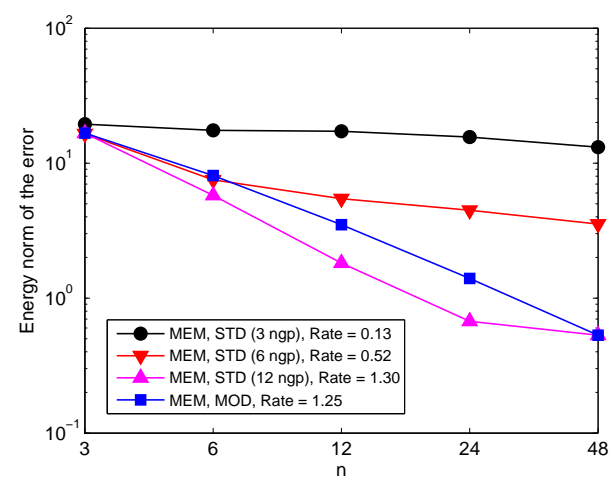

(c)

Fig. 14: Rate of convergence of norms for the colliding flow problem using standard and modified Gauss integration techniques. (a) $L^{2}$-norm of the velocity error, (b) $L^{2}$-norm of the pressure error; and (c) energy norm of the error. $n \times n$ ( $n$ is the number of divisions along the $x$ or $y$-directions) mesh pattern is used.

cavity flow and zero-velocity tests, one constant pressure mode was obtained in both cases. However, the constant pressure mode can be eliminated if one essential boundary condition is removed from the boundary. We also 


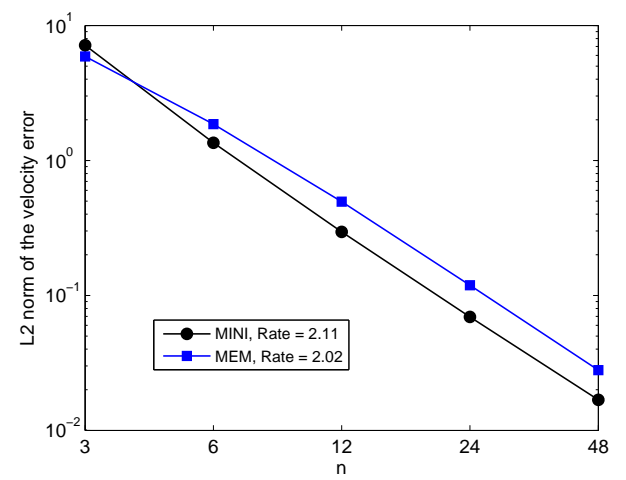

(a)

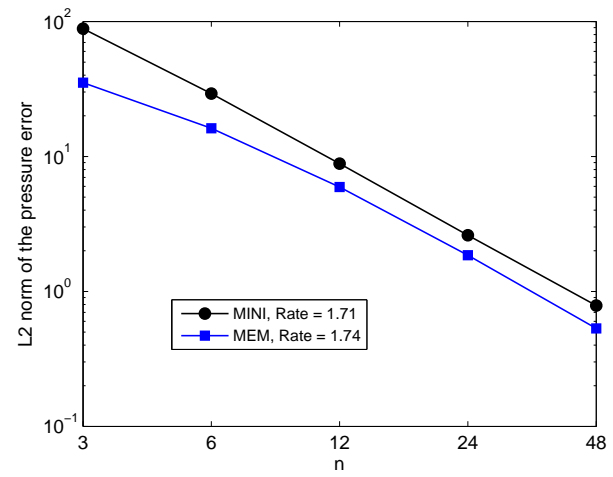

(b)

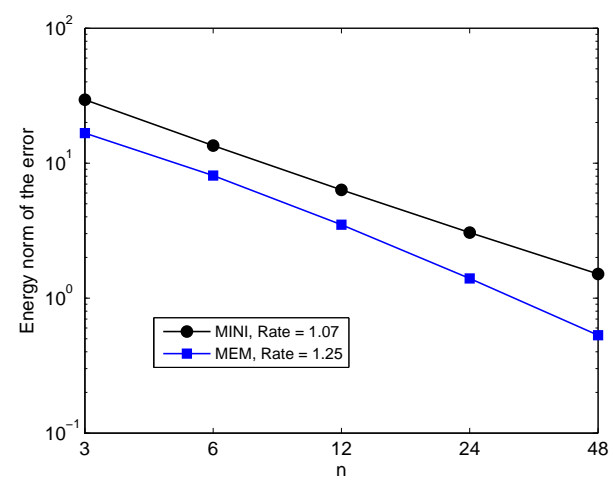

(c)

Fig. 15: Rate of convergence of norms for the colliding flow problem. (a) $L^{2}$-norm of the velocity error, (b) $L^{2}$-norm of the pressure error; and (c) energy norm of the error. $n \times n$ ( $n$ is the number of divisions along the $x$ or $y$-directions) mesh pattern is used.

mention that a zero-pressure field was obtained for the zero-velocity test, which indicates that the MEM formulation is free of spurious pressure modes. The inf-sup test is therefore passed and the MEM formulation is stable. 
Table 2: Values of $\alpha_{h}$ in the numerical inf-sup tests.

\begin{tabular}{|c|c|c|c|c|}
\hline Problem & $n=4$ & $n=8$ & $n=12$ & $n=16$ \\
\hline Cavity & 0.295 & 0.308 & 0.308 & 0.300 \\
Poiseuille & 0.112 & 0.113 & 0.113 & 0.113 \\
Zero-velocity & 0.295 & 0.308 & 0.308 & 0.300 \\
\hline
\end{tabular}

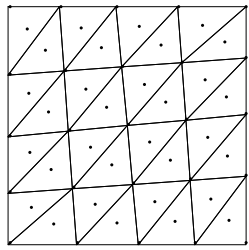

(a)

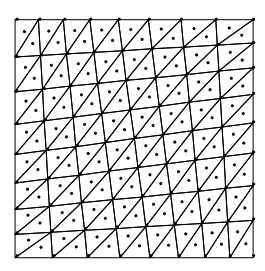

(b)

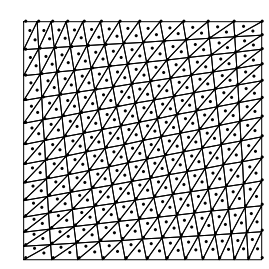

(c)

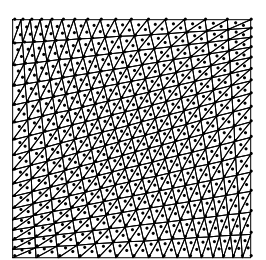

(d)

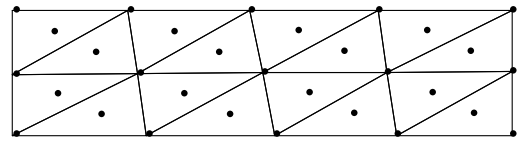

(e)

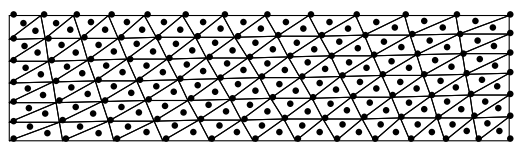

(g)

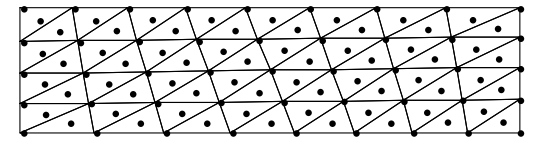

(f)

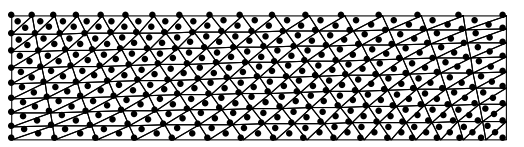

(h)

Fig. 16: Distorted background meshes employed for the inf-sup test. (a), (b), (c) and (d) for the leaky-lid driven cavity flow and the zero-boundary velocity problems; (e), (f), (g) and (h) for the Poiseuille problem. $n \times n$ mesh pattern is used for the leaky-lid driven cavity flow and zero-boundary velocity problems, while for the Poiseuille flow, $n \times n / 2$ mesh pattern is considered. In both cases $n$ is the number of divisions along the $x$-direction. 


\section{Concluding Remarks}

In this paper, a meshfree method based on maximum-entropy approximants that we recently presented in Ref. [1] was extended to Stokes flow in two dimensions and to three-dimensional analysis of incompressible linear elastic solids. The adoption of max-ent basis functions provides flexibility and eases the implementation since it permits the direct imposition of essential boundary conditions. A standard $u-p$ mixed formulation was used to compute volume-averaged nodal pressures a posteriori from the displacement field of surrounding nodes, which led to a single-field ( $u$-based) formulation. Various benchmark problems in two and three dimensions, which included a three-dimensional cantilever beam, a three-dimensional rigid flat punch problem, a leaky-lid driven cavity flow, and a colliding flow over a square domain were conducted to demonstrate the performance of the maximum-entropy meshfree method for incompressible media problems. The three-dimensional problems were tested on unstructured tetrahedral integration meshes and excellent accuracy was realized by the MEM method. For the three-dimensional cantilever beam problem, we found that accuracy considerations demanded the need for very high-order Gauss quadrature rule on unstructured meshes, whereas second-order accuracy sufficed for the numerical integration of both the volume and surface integrals in our modified integration scheme. On the benchmark problems, good agreement with analytical and reference solution results was found, and the MEM solution delivered the optimal rate of convergence in energy- and $L^{2}$-norms. We studied the stability of our method by conducting numerical inf-sup test on three benchmark problems. With nodal refinement, the numerical inf-sup value remained a constant that 
was bounded away from zero; furthermore, there were no spurious pressure modes. The accuracy and robustness of the MEM method in two- and threedimensional incompressible linear problems suggests its potential in threedimensional nonlinear simulations.

\section{ACKNOWLEDGEMENT}

The authors (A. Ortiz and N. Sukumar) gratefully acknowledge the research support of the National Science Foundation through contract Grants CMMI-0626481 and CMMI-0826513 to the University of California at Davis. A. Ortiz also thanks Professor K. J. Bathe for helpful clarifications on the inf-sup test. The work of M. A. Puso was performed under the auspices of the U.S. Department of Energy by Lawrence Livermore National Laboratory under Contract DE-AC52-07NA27344.

\section{References}

[1] A. Ortiz, M. A. Puso, N. Sukumar, Maximum-entropy meshfree method for compressible and near-incompressible elasticity, Computer Methods in Applied Mechanics and Engineering 199 (25-28) (2010) 1859-1871.

[2] F. Brezzi, On the existence, uniqueness and approximation of saddlepoint problems arising from Lagrangian multipliers, RAIRO, Analyse Numérique 8 (1974) 129-151.

[3] D. S. Malkus, T. J. R. Hughes, Mixed finite element methods - reduced and selective integration techniques: a unification of concepts, Computer Methods in Applied Mechanics and Engineering 15 (1) (1978) 63-81. 
[4] O. A. Ladyzhenskaya, The Mathematical Theory of Viscous Incompressible Flows, Gordon and Breach, London, 1969.

[5] I. Babuška, The finite element method with Lagrangian multipliers, Numerische Mathematik 20 (3) (1973) 179-192.

[6] K. J. Bathe, Finite Element Procedures, Prentice Hall, Englewood Cliffs, NJ, 1996.

[7] D. Chapelle, K. J. Bathe, The inf-sup test, Computers and Structures $47(4-5)(1993)$ 537-545.

[8] F. Brezzi, J. Pitkäranta, On the stabilization of finite element approximations of the Stokes equations, In: W. Hackbusch (Ed.), Efficient solutions of elliptic systems. Notes on numerical fluid mechanics 10 (1984) $11-19$.

[9] T. J. R. Hughes, L. P. Franca, M. Balestra, A new finite element formulation for computational fluid dynamics: V. Circumventing the BabuškaBrezzi condition: a stable Petrov-Galerkin formulation of the Stokes problem accommodating equal-order interpolations, Computer Methods in Applied Mechanics and Engineering 59 (1) (1986) 85-99.

[10] K. J. Bathe, The inf-sup condition and its evaluation for mixed finite element methods, Computers and Structures 79 (9) (2001) 243-252.

[11] F. Brezzi, M. Fortin, Mixed and Hybrid Finite Element Methods, Springer, NY, 1991. 
[12] D. Boffi, F. Brezzi, L. F. Demkowicz, R. G. Durn, R. S. Falk, M. Fortin, Mixed Finite Elements, Compatibility Conditions, and Applications. Edited by D. Boffi and L. Gastaldi. Lecture Notes in Mathematics, 1939, Springer-Verlag, Berlin Fondazione C.I.M.E., Florence, 2008.

[13] J. Li, H. Yinnian, Z. Chen, Performance of several stabilized finite element methods for the Stokes equations based on the lowest equal-order pairs, Computing 86 (1) (2009) 37-51.

[14] J. Dolbow, T. Belytschko, Volumetric locking in the element free Galerkin method, International Journal for Numerical Methods in Engineering 46 (6) (1999) 925-942.

[15] S. De, K. J. Bathe, Displacement/pressure mixed interpolation in the method of finite spheres, International Journal for Numerical Methods in Engineering 51 (3) (2001) 275-292.

[16] D. González, E. Cueto, M. Doblaré, Volumetric locking in natural neighbour Galerkin methods, International Journal for Numerical Methods in Engineering 61 (4) (2004) 611-632.

[17] J. Yvonnet, P. Villon, F. Chinesta, Natural element approximations involving bubbles for treating mechanical models in incompressible media, International Journal for Numerical Methods in Engineering 66 (7) (2006) 1125-1152.

[18] H. Desimone, S. Urquiza, H. Arrieta, E. Pardo, Solution of Stokes equations by moving least squares, Communications in Numerical Methods in Engineering 14 (10) (1998) 907-920. 
[19] X. L. Li, J. L. Zhu, A meshless Galerkin method for Stokes problems using boundary integral equations, Computer Methods in Applied Mechanics and Engineering 198 (34-40) (2009) 2874-2885.

[20] X. L. Li, Meshless analysis of two-dimensional Stokes flows with the Galerkin boundary node method, Engineering Analysis with Boundary Elements 34 (1) (2010) 79-91.

[21] A. Huerta, Y. Vidal, P. Villon, Pseudo-divergence-free element free Galerkin method for incompressible fluid flow, Computer Methods in Applied Mechanics and Engineering 193 (12-14) (2004) 1119-1136.

[22] D. L. Young, S. C. Jane, C. Y. Lin, C. L. Chiu, K. C. Chen, Solutions of 2D and 3D Stokes laws using multiquadrics method, Engineering Analysis with Boundary Elements 28 (10) (2004) 1233-1243.

[23] M. H. Mohammadi, Stabilized meshless local Petrov-Galerkin (MLPG) method for incompressible viscous fluid flows, CMES: Computer Modeling in Engineering \& Sciences 29 (2) (2008) 75-94.

[24] M. Cheng, G. R. Liu, A novel finite point method for flow simulation, International Journal for Numerical Methods in Fluids 39 (12) (2002) $1161-1178$.

[25] M. B. Liu, W. P. Xie, G. R. Liu, Modeling incompressible flows using a finite particle method, Applied Mathematical Modelling 29 (12) (2005) $1252-1270$.

[26] J. Fang, A. Parriaux, A regularized Lagrangian finite point method for 
the simulation of incompressible viscous flows, Journal of Computational Physics 227 (20) (2008) 8894-8908.

[27] H. Wendland, Divergence-free kernel methods for approximating the Stokes problem, SIAM Journal on Numerical Analysis 47 (4) (2009) 3158-3179.

[28] T. P. Fries, H. G. Matthies, A stabilized and coupled meshfree/meshbased method for the incompressible Navier-Stokes equationsPart I: Stabilization, Computer Methods in Applied Mechanics and Engineering 195 (44-47) (2006) 6205-6224.

[29] X. Li, Q. Duan, Meshfree iterative stabilized Taylor-Galerkin and characteristic-based split (CBS) algorithms for incompressible NS equations, Computer Methods in Applied Mechanics and Engineering 195 (44-47) (2006) 6125-6145.

[30] L. Zhang, J. Ouyang, X. H. Zhang, W. B. Zhang, On a multi-scale element-free Galerkin method for the Stokes problem, Applied Mathematics and Computation 203 (2) (2008) 745-753.

[31] J. Dolbow, T. Belytschko, Numerical integration of Galerkin weak form in meshfree methods, Computational Mechanics 23 (3) (1999) 219-230.

[32] I. Babuška, U. Banerjee, J. E. Osborn, Q. L. Li, Quadrature for meshless methods, International Journal for Numerical Methods in Engineering 76 (9) (2008) 1434-1470.

[33] E. T. Jaynes, Information theory and statistical mechanics, Physical Review 106 (4) (1957) 620-630. 
[34] N. Sukumar, Construction of polygonal interpolants: a maximum entropy approach, International Journal for Numerical Methods in Engineering 61 (12) (2004) 2159-2181.

[35] M. Arroyo, M. Ortiz, Local maximum-entropy approximation schemes: a seamless bridge between finite elements and meshfree methods, International Journal for Numerical Methods in Engineering 65 (13) (2006) $2167-2202$.

[36] N. Sukumar, R. W. Wright, Overview and construction of meshfree basis functions: from moving least squares to entropy approximants, International Journal for Numerical Methods in Engineering 70 (2) (2007) 181-205.

[37] S. Li, W. K. Liu, Meshfree and particle methods and their applications, Applied Mechanics Reviews 55 (1) (2002) 1-34.

[38] T. P. Fries, H. G. Matthies, Classification and overview of meshfree methods, Tech. Rep. Informatikbericht-Nr. 2003-03, Institute of Scientific Computing, Technical University Braunschweig, Braunschweig, Germany (2004).

[39] S. Fernández-Méndez, A. Huerta, Imposing essential boundary conditions in mesh-free methods, Computer Methods in Applied Mechanics and Engineering 193 (12-14) (2004) 1257-1275.

[40] L. L. Yaw, N. Sukumar, S. K. Kunnath, Meshfree co-rotational formulation for two-dimensional continua, International Journal for Numerical Methods in Engineering 79 (8) (2009) 979-1003. 
[41] A. M. Rosolen, R. D. Millán, M. Arroyo, On the optimum support size in meshfree methods: a variational adaptivity approach with maximum entropy approximants, International Journal for Numerical Methods in Engineering 82 (7) (2010) 868-895.

[42] T. J. R. Hughes, The Finite Element Method: Linear Static and Dynamic Finite Element Analysis, Dover Publications, Inc, Mineola, NY, 2000.

[43] J. Bonet, A. J. Burton, A simple average nodal pressure tetrahedral element for incompressible and nearly incompressible dynamic explicit applications, Communications in Numerical Methods in Engineering 14 (5) (1998) 437-449.

[44] C. R. Dohrmann, M. W. Heinstein, J. Jung, S. W. Key, W. R. Witkowski, Node-based uniform strain elements for three-node triangular and four-node tetrahedral meshes, International Journal for $\mathrm{Nu}-$ merical Methods in Engineering 47 (9) (2000) 1549-1568.

[45] M. A. Puso, J. Solberg, A stabilized nodally integrated tetrahedral, International Journal for Numerical Methods in Engineering 67 (6) (2006) 841-867.

[46] P. Krysl, B. Zhu, Locking-free continuum displacement finite elements with nodal integration, International Journal for Numerical Methods in Engineering 76 (7) (2008) 1020-1043.

[47] D. N. Arnold, F. Brezzi, M. Fortin, A stable finite element for the Stokes equations, Calcolo 21 (4) (1984) 337-344. 
[48] J. C. Simo, T. J. R. Hughes, On the variational foundations of assumed strain methods, Journal of Applied Mechanics 53 (1) (1986) 51-54.

[49] M. A. Puso, J. S. Chen, E. Zywicz, W. Elmer, Meshfree and finite element nodal integration methods, International Journal for Numerical Methods in Engineering 74 (3) (2008) 416-446.

[50] F. Brezzi, K. J. Bathe, A discourse on the stability conditions for mixed finite element formulations, Computer Methods in Applied Mechanics and Engineering $82(1-3)(1990) 27-57$.

[51] R. L. Taylor, A mixed-enhanced formulation for tetrahedral finite elements, International Journal for Numerical Methods in Engineering $47(1-3)(2000)$ 205-227.

[52] J. Bonet, M. Marriot, O. Hassan, An averaged nodal deformation gradient linear tetrahedral element for large strain explicit dynamic applications, Communications in Numerical Methods in Engineering 17 (8) (2001) 551-561.

[53] F. M. Andrade Pires, E. A. de Souza Neto, J. L. de la Cuesta Padilla, An assessment of the average nodal volume formulation for the analysis of nearly incompressible solids under finite strains, Communications in Numerical Methods in Engineering 20 (7) (2004) 569-583.

[54] G. Irving, C. Schroeder, R. Fedkiw, Volume conserving finite element simulations of deformable models, ACM Transactions on Graphics 26 (3) (2007) 13.1-13.6. 
[55] E. A. de Souza Neto, F. M. Andrade Pires, D. R. J. Owen, F-bar-based linear triangles and tetrahedra for finite strain analysis of nearly incompressible solids. Part I: formulation and benchmarking, International Journal for Numerical Methods in Engineering 62 (3) (2005) 353-383.

[56] P. Hauret, E. Kuhl, M. Ortiz, Diamond elements: a finite element/discrete-mechanics approximation scheme with guaranteed optimal convergence in incompressible elasticity, International Journal for Numerical Methods in Engineering 72 (3) (2007) 253-294.

[57] N. Kikuchi, Remarks on 4CST-elements for incompressible materials, Computer Methods in Applied Mechanics and Engineering 37 (1) (1983) $109-123$.

[58] O. C. Zienkiewicz, R. L. Taylor, The Finite Element Method, Volume 1: The Basis, 5th Edition, Butterworth-Heinemann, Oxford, UK, 2000.

[59] K. B. Nakshatrala, A. Masud, K. D. Hjelmstad, On finite element formulations for nearly incompressible linear elasticity, Computational Mechanics 41 (4) (2008) 547-561.

[60] D. Z. Turner, K. B. Nakshatrala, K. D. Hjelmstad, On the stability of bubble functions and a stabilized mixed finite element formulation for the Stokes problem, International Journal for Numerical Methods in Fluids 60 (12) (2009) 1291-1314.

[61] P. Hansbo, M. G. Larson, Piecewise divergence-free discontinuous Galerkin methods for Stokes flow, Communications in Numerical Methods in Engineering 24 (5) (2008) 355-366. 
[62] H. C. Elman, D. J. Silvester, A. J. Wathen, Finite Elements and Fast Iterative Solvers: with Applications in Incompressible Fluid Dynamics, Oxford University Press, Inc, NY, 2006. 